\title{
A Study of Extended Spectrum Beta-Lactamase (ESBL) Producing Gram Negative Uropathogens and their Antimicrobial Susceptibility Pattern in a Tertiary Care Hospital
}

\author{
S. Rajesh*, D. Neelaveni, N. Subathra and B. Divya \\ Department of Microbiology, Government Mohankumaramangalam Medical College, \\ Salem, India \\ *Corresponding author:
}

\section{A B S T R A C T}

Urinary tract infection (UTI) includes the infection of urethra, bladder, ureters and kidneys which comprise the urinary tract. UTI is an important cause of morbidity and mortality in both developing and developed countries of the world. Urine samples were collected from In-patients and Out-patients attending hospital with the history suggestive of Urinary Tract Infection. Clean catch mid-stream urine samples were collected in sterile universal containers after proper cleansing of urethra and perineum. 1200 samples were collected from IP/OP patients. The sample size was higher in number because we got more samples than what are expected approximately for two months while starting the study. These samples were processed within 2 hours of collection without delay. A total no of 1,200 samples were collected from in-patient and Out-patient departments of the tertiary care hospital. Out of these 1,200 samples significant bacteriuria (30\%) was observed in 354 samples. Out of these 354 isolates, 63(10.1\%) isolates were found to be Gram positive cocci, 290(81.9\%) were Gram negative bacilli and 1(0.2\%) fungi-Candida spp. Among total 290 GNB isolates E. coli 146(41.2\%), Klebsiella pneumoniae 81(22.8 \%), Klebsiella oxytoca 15 (4.2\%), Pseudomonas aeruginosa 20(5.6\%), Proteus spp 10(2.8\%), Acinetobacter spp 8(2.2\%), Citrobacter spp 6(1.6\%), Morganella spp 3(1\%), and Providencia spp 1(0.2\%) were isolated. Among the total GPC isolates Staphylococcus aureus 8(2.2\%), CONS 54(15.2\%) and Enterococcus spp 1(0.2\%) were isolated. ESBLs are now causing the major threatening infections in hospitalized patients throughout the world. The prevalence of ESBLs among clinical isolates varies greatly in worldwide. Major risk factors for colonization of ESBLs are long term antibiotic therapy, prolonged hospital stay and $\mathrm{H} / \mathrm{O}$ instrumentation or catheterization. In India the prevalence of ESBL production varies from $10 \%$ to $84 \%$. Cefoperazone/Sulbactum $98.5 \%$ and Piperacillin/Tazobactum 97\% are very effective in treatment of ESBL producing organism associated urinary tract infection. Amikacin, Nitrofurantoin followed by Gentamicin are showed higher susceptibility rate when compared to other drugs and these can be used as I line empirical drugs in UTI according to the patient's renal condition.

\section{Introduction}

Urinary tract infection (UTI) includes the infection of urethra, bladder, ureters and kidneys which comprise the urinary tract. UTI is an important cause of morbidity and mortality in both developing and developed countries of the world. UTI affects all age 
groups and both sexes and most UTI occur by the common route of infection i.e. ascending route after entry via the urethral meatus (Ahmed et al., 2014). Gram negative bacilli are the most common organism to cause UTI about $80-85 \%$ in both out-patients and inpatients. In the Gram Negative pathogens, $\beta$ lactamase production remains the most important contributing factor to $\beta$-lactam antibiotic resistance (Lakshminarayanan and Sangeetha, 2015). The major group of Beta lactamase enzymes that conferring resistance to oxyimino-cephalosporins are Extended spectrum Beta-lactamases (ESBL) (Alan Philpppon et al., 1989). These ESBL enzymes are plasmid mediated and belongs to Ambler class A and Bush- Jacoby-Medieros functional class 2be. By common definition, ESBLs are capable of conferring bacterial resistance to the Penicillins, Cephalosporins (I, II and III generation) and Aztreonam (but not to the Cephamycin and Carbapenem) by hydrolysis of these antibiotics, and which are inhibited by $\beta$-lactamase inhibitors such as Clavulanic acid, Sulbactum and Tazobactum (Shaikh et al., 2015; Chauhan et al., 2014).

ESBLs are the important factor for emerging infections nowadays because they are plasmid borne enzymes and have a potential role in transferring multiple drug resistance among different organisms and also from one patient to another (Supriya et al., 2004). Since it represents the epidemiological marker of colonization, detection and regular surveillance of ESBL producers from the sample such as urine is of utmost important (Paterson et al., 2004). The prevalence of ESBL producing organisms among clinical isolates vary greatly in worldwide and is steadily increasing over the various parts and it results in severe complications because of limited and ineffective antibiotic treatment. This will lead to major clinical crisis such as prolonged hospital stay, increased morbidity, mortality and health care costs to patients (Trupti Bajpai et al., 2014). Therefore to control the outbreak of ESBL producing organisms are considered as a clinically important and major concern now.

Hence the present study was designed to detect the ESBL production among Uropathogens in UTI patients by phenotypic methods and to assess the changes in antimicrobial susceptibility pattern for preparing antibiotic policy in our hospital.

Extended Spectrum Beta-lactamase (ESBL) isolates were first detected in Western Europe in mid-1980s. Since then their incidence have been increasing sharply and have become recognized as a major worldwide problem. ESBL producing organisms have been detected in every inhabited continent. Several workers from foreign and India including Rugved Lulkarni et al., (2013) of BJ Govt. Medical college (Pune), Tripti Bajpai et al., (2014) of IGNOU (New Delhi), Supriya et al., (2004) (Nagpur), Syed Muataq Ahmed et al., (2014) (Kerala), Chauhan et al., (2014) (Dehradun) and Lakshminarayana et al., (2015) (Bengaluru) had done researches based on ESBL producers and noted their sensitivity pattern in UTI.

David Paterson et al., (Pennsylvania), Amita Jain et al., (Luknow), Modi Dhara et al., (Ahmedabad) and Mita D Wadekar et al., (Mysore) were done the studies regarding detection of ESBL among gram negative bacilli from various samples. Similarly this study highlights the prevalence of ESBL producers and their antibiotic susceptibility pattern in a tertiary care hospital.

The main aim and objectives of this study includes to determine the Bacterial profile and their Anti-microbial susceptibility pattern of microbes in UTI patients and to detect and evaluate the prevalence of Extended Spectrum Beta Lactamase (ESBL) producing Gram Negative Uropathogens among the isolates. Also to determine the Antimicrobial 
susceptibility pattern for ESBL producers and ESBL non producers.

\section{Materials and Methods}

Type of study : Prospective study.

Place of study : Govt. Mohan Kumaramangalam Medical College Hospital, Salem.

Study period : 6 months (July to december2016)

Nature of sample: Urine

Sample size : 1200 samples

\section{Sample collection}

The study was carried out after getting the ethical committee approval and informed consent was obtained from each patient before sample collection. Urine samples were collected from In-patients and Out-patients attending hospital with the history suggestive of Urinary Tract Infection. Standard guidelines were followed for sample collection and processing. Clean catch midstream urine samples were collected in sterile universal containers after proper cleansing of urethra and perineum. 1200 samples were collected from IP/OP patients. The sample size was higher in number because we got more samples than what are expected approximately for two months while starting the study. These samples were processed within 2 hours of collection without delay.

\section{Sample processing method}

After receiving the samples, Gram staining (Picture 1) was done for all samples and then inoculated in Nutrient agar, MacConkey agar and 5\% sheep Blood agar. For inoculation, semi-quantitative plating method was adapted by using a calibrated loop delivering $0.001 \mathrm{ml}$ of urine. The plates were incubated at $37^{\circ} \mathrm{C}$ for $24-48$ hours. After incubation, the bacterial isolates were identified based on the colony characteristics. Only the isolates correlate with Direct Gram staining and with colony count $>10^{5} \mathrm{CFU} / \mathrm{ml}$ (significant bacteriuria) and $10^{4} \mathrm{CFU} / \mathrm{ml}$ (according to patient's condition) were considered for further processing. All confirmed isolates were further speciated by standard biochemical test protocols (Bailey and Scott's Diagnostic Microbiology, 2007. XII edn).

The isolates were tested for Anti-microbial susceptibility by Modified Kirby Bauer Discdiffusion method with commercially available discs (Hi-media) on Mueller Hinton agar plates and the results were interpreted according to CLSI guidelines using Escherichia coli ATCC 25922 as a control strain (Kulkarni et al., 2013).

The panel of antibiotic discs like Ampicillin $(10 \mu \mathrm{g})$, Amikacin $(30 \mu \mathrm{g})$, Gentamicin $(15 \mu \mathrm{g})$, Cefotaxime $(30 \mu \mathrm{g}), \quad$ Ceftazidime $(30 \mu \mathrm{g})$ Cotrimoxazole $(25 \mu \mathrm{g})$, Ciprofloxacin $(5 \mu \mathrm{g})$, Norfloxacin $(10 \mu \mathrm{g})$ and Nitrofurantoin $(300 \mu \mathrm{g})$ were used as first line drugs and Cefoperazone/Sulbactum (30/10 mcg), Piperacillin/Tazobactum (100/10mcg) and Imipenem $(10 \mathrm{mcg})$ were used as second line drugs.

An isolate has been considered as multi drug resistant (picture 2) if it is resistant to 3 or more than 3 Antimicrobial agents. Relevant demographic data is also collected.

\section{Screening for ESBL}

After Antimicrobial susceptibility test, isolates which have zone of inhibition $\leq$ $27 \mathrm{~mm}$ for cefotaxime and $\leq 22 \mathrm{~mm}$ for Ceftazidime are considered as presumptive ESBL producers and these isolates were subjected to phenotypic tests such as Combination Disc Test (CDT) and Double Disc Synergy Test (DDST) (Kulkarni et al., 2013). 
Phenotypic confirmation methods for ESBL

All ESBL detection and confirmation was done according to CLSI (2014) guidelines using E. coli ATCC 25922 as negative control and Klebsiella pneumoniae ATCC 700603 as positive control.

\section{Combination Disc Test (CDT) [Combined Disc Test]}

For each test, inoculum was prepared and the turbidity was adjusted to 0.5 McFarland standard. The organism was swabbed on the Mueller Hinton agar plate.

Discs containing Ceftazidime $(30 \mu \mathrm{g})$ alone and combined with clavulanic acid $(10 \mu \mathrm{g})$ were placed at a distance (edge to edge) of $20 \mathrm{~mm}$ in the centre of plate. After incubation, the positive result was indicated when the difference in zone of inhibition (picture 3) around the Ceftazidime/clavulanic acid disc and Ceftazidime disc alone was $\geq 5 \mathrm{~mm}$ (Jain et al., 2003; Modi Dhara et al., 2012).

\section{Double Disc Synergy Test (DDST)}

For each test, inoculum was prepared and the turbidity was adjusted to 0.5 McFarland standard and the inoculum was swabbed on the Mueller Hinton agar plate. The disc containing Amoxicillin (20 $\mu \mathrm{g}) /$ Clavulanic acid $(10 \mu \mathrm{g})$ was placed in the centre of the agar plate.

Third generation Cephalosporins like disc containing Cefotaxime $(30 \mu \mathrm{g})$, Ceftazidime $(30 \mu \mathrm{g})$ and Ceftriaxone $(30 \mu \mathrm{g})$ were applied to the periphery of Amox/Clav disc (centre to centre in position). The distances between the discs (edge to edge) were adjusted to be 15$20 \mathrm{~mm}$. Positive result was indicated when the inhibition zones around any of the cephalosporin discs are augmented towards the direction of disc containing clavulanic acid (Modi Dhara et al., 2012; Liofilchem, 2014).

\section{Results and Discussion}

A total no of 1,200 samples were collected from in-patient and Out-patient departments of the tertiary care hospital. Out of these 1,200 samples significant bacteriuria $(30 \%)$ was observed in 354 samples. Out of these 354 isolates, 63(10.1\%) isolates were found to be Gram positive cocci, 290(81.9\%) were Gram negative bacilli and 1(0.2\%) fungiCandida spp. Among total 290 GNB isolates E. coli 146(41.2\%), Klebsiella pneumoniae 81(22.8\%), Klebsiella oxytoca 15 (4.2\%), Pseudomonas aeruginosa 20(5.6\%), Proteus spp 10(2.8\%), Acinetobacter spp 8(2.2\%), Citrobacter spp 6(1.6\%) Morganella spp $3(1 \%)$, and Providencia spp 1(0.2\%) were isolated. Among the total GPC isolates Staphylococcus aureus 8(2.2\%), CONS 54(15.2\%) and Enterococcus spp 1(0.2\%) were isolated.

Among 290 GNB isolates 160(55\%) isolates were found to be Multi drug resistant. $153(52.7 \%)$ were resistant to Cefotaxime, 162(56\%) were resistant to Cefotaxime and Ceftazidime and 162(56\%) were found to be presumptive ESBL producers. Those were subjected to confirmation test by Combination Disc Test (CDT) and Double Disc Synergy Test (DDST).

After the confirmation test, Combination Disc test yield positive results for $134(46 \%)$ isolates and $115(40 \%)$ by Double Disc Synergy test and isolates confirmed as ESBL producers by both phenotypic methods were $115(40 \%)$ [Table-1].

Of those ESBL (phenotypic confirmed) producers $79 \%$ were susceptible to Amikacin, $52 \%$ to Gentamicin, $73 \%$ to Nitrofurantoin, 
$32 \%$ to Ciprofloxacin, $18 \%$ to Cotrimoxazole, $15 \%$ to Norfloxacin, $9 \%$ to Ampicillin, 98.5\% to Cefoperazone/Sulbactum and $97 \%$ to Piperacillin/Tazobactum.

While the non-ESBL producers were $86 \%$ susceptible to Amikacin, $74 \%$ to Nitrofurantoin, $51 \%$ to Gentamicin, $45 \%$ to Cotrimoxazole $44 \%$ to Ciprofloxacin, and $36 \%$ to Norfloxacin, $11 \%$ to Ampicillin, $100 \%$ Cefoperazone/Sulbactum, $100 \%$ to Piperacillin/Tazobactum. All GNB isolates were showed $100 \%$ sensitivity to Imipenem [Chart-2].

Detection of extended spectrum Betalactamase producing Uropathogens are an important marker of endemicity. In our study 290 (81.9\%) negative bacilli were isolated among the total isolates. E. coli was the most common isolate (41.2\%) followed by Klebsiella $\operatorname{spp}(27.1 \%)$. This is similar to Rugved Kulkarni et al., (2013) (E. coli29.1\%, Klebsiella spp-7.9\%), Lakshminarayana et al., (2015) (E. coli65.9\%, Klebsiella spp-15.4\%) and Trupti Bajpai et al., (2014) (E. coli-42.7\%, Klebsiella spp, 17.7\%) studies.

Our study culture positivity is higher in IPD (87\%) samples as compared to OPD (12.9\%) samples. This is supported by Trupti Bajpai (2014) et al., (IPD-42.1\%, OPD-30\%) and Rugved Kulkarni et al., (2013) (IPD-89.7\%, OPD-10.2\%) and Lakshminarayana (2015) et al., (IPD-53.1\%, OPD-46.8\%) studies.

In our study, ESBL producers were more prevalent in female $(55.08 \%)$ than male $(44.9 \%)$ patients. This supported by Lakshminarayana et al., (2015) (female$59.9 \%$, male-40.09\%) study. In contrast Trupti Bajpai et al., (2014) study showed more isolates from male $(39.8 \%)$ than female $(36.6 \%)$ patients. This may be due to number of samples and other co-morbid conditions were high in female patients in our study.
ESBLs are more prevalent in Escherichia coli $21.7 \%$ followed by Klebsiella spp $13.4 \%$ in our study. This report is correlated with Babypadmini et al., (2004) study in which ESBL production was found to be $41 \%$ in $E$. coli and $40 \%$ in Klebsiella spp. In contrast Supriya et al., (2004) study showed Klebsiella spp $(25.6 \%)$ followed by E. coli $(18.5 \%)$, Lakshminarayana et al., (2015) study E. coli $35.1 \%$ followed by Citrobacter spp 30\%, Trupti Bajpai et al., (2014) study E. coli 41.6\% followed by Pseudomonas aeruginosa $36.1 \%$ and in Rugved kukarni et al., (2013) study showed E. coli $40.7 \%$ followed by Proteus spp $25.7 \%$.

The present study showed that the Double disk synergy test has less sensitivity than Combination disk test (DDST-40\%, CDT46\%) which is similar to Mita et al., study and it may be due to storage temperature of discs and problem of optimal disc space (Wadekar et al., 2013).

In our study ESBL producers were showed high susceptible rate to first line drugs like Amikacin 79\%, Nitrofurantoin $73 \%$ followed by Gentamicin $52 \%$ and showed more than 95\% susceptibility to second line drugs like Cefoperazone/Sulbactum and Piperacillin/Tazobactum. All ESBL producers were showed $100 \%$ susceptibility to Imipenem. This first line drug susceptibility pattern is supported by Lakshminarayana et al., (2015) (Amikacin 88.3\%, Nitrofurantoin 84.6\%) and Rugved kukarni et al., (2013) (Amikacin 70.4\%, Nitrofurantoin 75\%) studies.

In our study non ESBL producers were showed high susceptible rate to first line drugs like Amikacin 86\%, Gentamicin 74\% followed by Nitrofurantoin 51\%. In contrast Rugved kukarni et al., (2013) study showed Amikacin $47.8 \%$, Gentamicin 27.4\% followed by Nitrofurantoin $26.8 \%$. 
ESBL producers showed susceptible rate to Cotrimoxazole $18 \%$ and Norfloxacin $15 \%$. This is similar to Trupti Bajpai et al., (2014) (Cotrimoxazole 12\% and Norfloxacin 17.4\%). Our study showed high degree of susceptible rate to Aminoglycosides (Amikacin 79\%,
$86 \%$ and Gentamicin 52\%, 74\%) for ESBL and non ESBL producers respectively. This is supported by Trupti Bajpai et al., (2014) (Amikacin 87.5\%, 63.2\% and Gentamicin $68.7 \%, \quad 50.5 \%) \quad$ study.

Table.1 Percentage of ESBL producers among GNB

\begin{tabular}{|l|c|c|c|}
\hline No of ESBL producers by & No of ESBL producers by phenotypic confirmation tests \\
screening test (Isolate & CDT & DDST & Both test \\
resistant to Ceftazidime and & & & \\
Cefotaxime ) & & $115(40 \%)$ & $115(40 \%)$ \\
\hline \multicolumn{2}{|c|}{$162(56 \%)$} & $134(46 \%)$ & \\
\hline
\end{tabular}

Picture.1 Gram staining showing pus cells with Gram negative bacilli

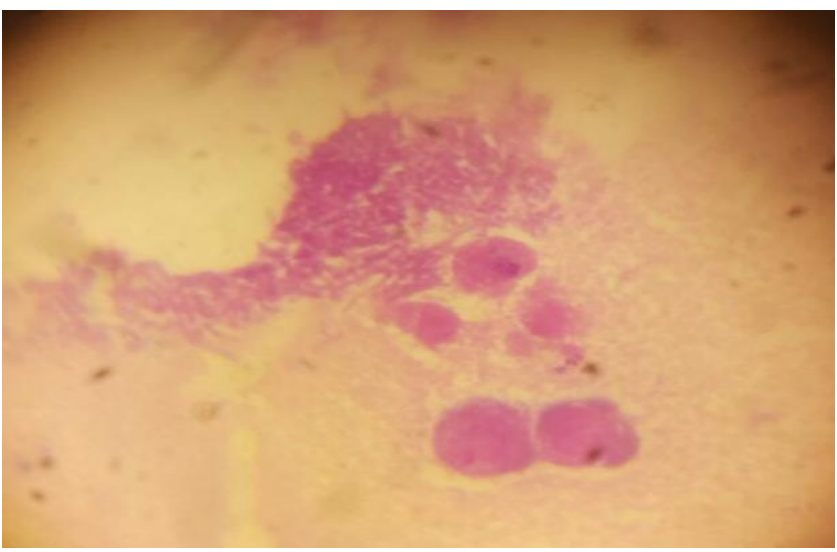

Picture.2 Antibiotic susceptibility test shows Multi drug resistant isolate

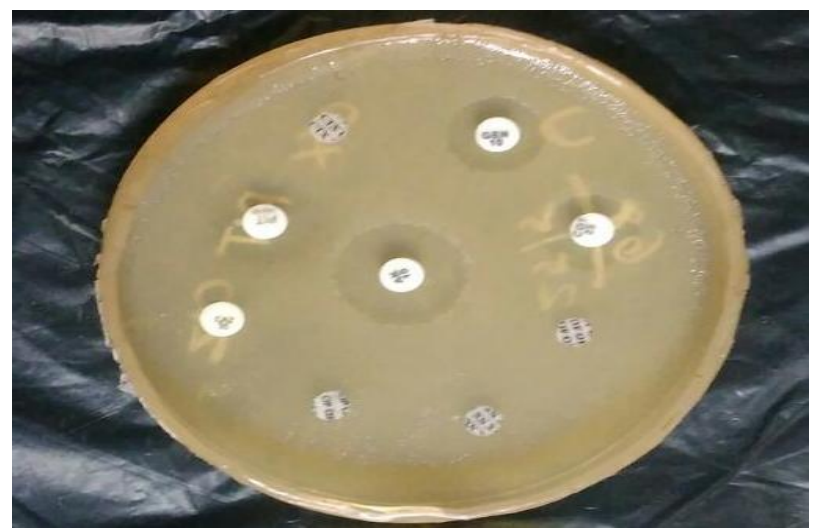


Picture.3 Combined Disc test showing difference in zone of inhibition

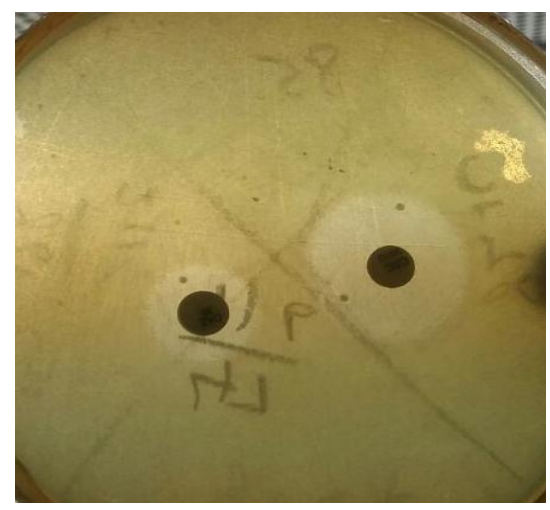

Chart.1 Distribution of pathogens in UTI patients

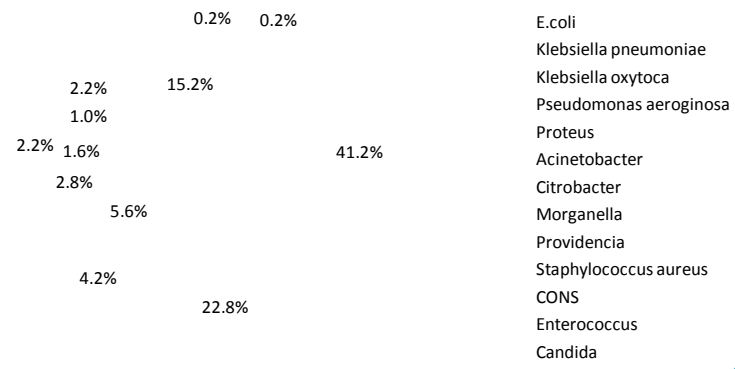

Chart.2 Antibiotic Susceptibility pattern for ESBL and non ESBL producers
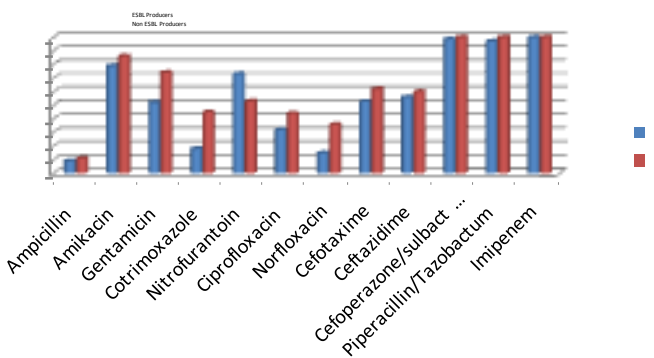
ESBLs are now causing the major threatening infections in hospitalized patients throughout the world. The prevalence of ESBLs among clinical isolates varies greatly in worldwide. Major risk factors for colonization of ESBLs are long term antibiotic therapy, prolonged hospital stay and $\mathrm{H} / \mathrm{O}$ instrumentation or catheterization. In India the prevalence of ESBL production varies from $10 \%$ to $84 \%$. Cefoperazone/Sulbactum $\quad 98.5 \%$ and Piperacillin/Tazobactum $97 \%$ are very effective in treatment of ESBL producing organism associated urinary tract infection.

Amikacin, Nitrofurantoin followed by Gentamicin are showed higher susceptibility rate when compared to other drugs and these can be used as I line empirical drugs in UTI according to the patient's renal condition.

The present study concludes that ESBL production was high among Uropathogens (50to 60\%) and most of the ESBL producers were multi drug resistant (50to 60\%). UTI caused by this multi drug resistance organisms are not able to treat by routine oral antibiotics like norfloxacin and ofloxacin and injectable cefotaxime like antibiotics.

Due to delay in diagnosis and delay in treatment with appropriate antibiotics in UTI which lead to septicaemia and pylonephritis like complication can occur. Phenotypic detection of ESBLs in routine antibiotic susceptibility testing and monitoring the continuous surveillance of ESBL detection and their susceptibility patterns are helpful to prevent treatment failure and formulation of appropriate antibiotic policy for UTI.

Early diagnosis of ESBL and treatment with appropriate antibiotics in urinary tract infection can control the spread of antibacterial resistance enzymes among patients and also we can prevent the hospital acquired infections.

\section{References}

Alan Philpppon, Rogrer Lbia, George Jacoby. Extended -Spectrum Beta Lactamses - Minireview. Antimicrobial agents and Chemotherapy, Aug 1989.p. 1131-1136.

Amita Jain, Indranil Roy, Mahendra K.Gupta, Mala Kumar and S.K.Agarwal. Prevalence of extended spectrum beta lactamases producing Gram negative bacteria in septicaemic neonates in a tertiary care hospital. Journal $\mathrm{f}$ Medical Microbiology (2003), 52, 421-425.

Babypadmini S, Appalaraju B. extended spectrum-betalactamases in urinary isolates of Escherichia coli and Klebsiella pneumoniae - prevalence and susceptibility pattern in a tertiary care hospital. Indian $\mathbf{J}$ med microbiology 2004; 22:172-4.

Bailey and Scott's Diagnostic Microbiology. 2007. XII edn. (p: 120-146, 172 214,340-349,629-709, 838 -39).

Chauhan S, Khanduri A, Mahawal.B.S. Extended Spectrum Beta Lactamses detection using two phenotypic methods among Uropathogens Escherichia coli. OQSR Journal of Dental and Medical Sciences. Vol 13, issue 4 Ver. VL.(Apr.2014), pp84-86.

CLSI M100-S24 - Performance Standards fir Antimicrobial Susceptibility testing, 2014.

David L Paterson, Wen-Chien Ko, Anne Von Gottherg. Antibiotic therapy for Klebsiella pneumoniae bacterimia: Implications of production of Extended-Spectrum beta lactamases. Clinical Infectious Diseases. 2004:39(1July)-31.

Koneman's color Atlas and Text book of microbiology. 2006, Sixth Ed.p (67110,141-160,303-350,945-1021, 11511243). 
Lakshminarayanan.S.A, Sangeetha.S Detection of ESBL producing Gram Negative Uropathogens and their antibiotic resistance pattern in tertiary care hospital, Bangalore, India.Int.J.Curr.Microbiol.App.Sci (2015) 4(12): 578-583.

Liofilchem- ESBL Disc testsRev2/19.06.2014.

Mita D.Wadekar, K.Anuradha and D.Venkatesha. Phenotypic detection of ESBL nad MBL in clinical isolates of Enterobacteriaceae. Int.J.Curr.Res.Aca.Rev.2013; 1(3): 89-95.

Modi Dhara, Patel Disha, Patel Sachin, Jain Manisha. Comparison of various methods for the detection of extended spectrum beta lactamases in Klebsiella pneumoniae isolated from neonatal intensive care unit, Ahmedabad. National Journal Medical Research. Vol 2/ issue 3/july-sept 2012.

Rugved Kulkarni, Vaishali Dohe,Dnyaneshwari Ghadge, Arvind Bhore. A study of extended spectrum Betalactamase (esbl) producers in clinical isolates. Medical journal of western India. vol41/issue 1/ feb 2013. Sibhahatulla Shaikh, Jamale Fatima, Shazi Shakil, Syed Mohd.Danish Rizvi,
Mohammad Amjad Kamal. Antibiotic resistance and extended spectrum beta-lactamases: Types, epidemiology and treatment. Saudi Journal of Biological Sciences (2015) 22, 99101.

Supriya S. Tankhiwale, Suresh V. Jalgaonkar, Sarfraz Ahamad and Umesh Hassani. Evaluation of extended spectrum beta lactamase in urinary isolates. Indian $\mathbf{J}$ Med Res 120, December 2004, pp553556.

Syed Mustaq Ahmed, sumita Rajeevan, P.T.Jasmin and V.P.A.Shakir. Detection of ESBL among the Gram negative Uropathogens and their antibiotic resistance pattern in arural medical college. Int.J.Curr.Microbiolo.App.Sci (2014) 3(2: 561-567.

Trupti Bajpai, Maneesha Pandey1, Meena Varma2, ganesh S. bhatambare Prevalence of extended spectrum betalactamase producing uropathogens and their antibiotic resistance profile in patients visiting a tertiary care hospital in central India: Implications on empiric therapy - Indian Journal of Pathology and Microbiology - 57(3), July-Sept 2014.

\section{How to cite this article:}

Rajesh, S., D. Neelaveni, N. Subathra and Divya, B. 2017. A Study of Extended Spectrum Beta-Lactamase (ESBL) Producing Gram Negative Uropathogens and their Antimicrobial Susceptibility Pattern in a Tertiary Care Hospital. Int.J.Curr.Microbiol.App.Sci. 6(5): 27222730. doi: https://doi.org/10.20546/ijcmas.2017.605.305 